\title{
Rotura cardíaca após infarto agudo do miocárdio (IAM): uma complicação passível de correção cirúrgica?
}

Luís Alberto DALLAN*, Sérgio Almeida de OLIVEIRA*, Carlos ABREU FILHO*, Richard H. CABRAL*, Fábio B. JATENE* ${ }^{\star}$, Paulo M. PÊGO-FERNANDES ${ }^{\star}$, José Carlos R. IGLÉSIAS ${ }^{\star}$, Marcelo B. JATENE* Geraldo VERGINELLI* ${ }^{*}$ Adib D. JATENE*

RBCCV 44205-224

DALLAN, L. A.; OLIVEIRA, S. A.; ABREU FILHO, C.; CABRAL, R. H.; JATENE, F. B.; PÊGO-FERNANDES, P. M.; IGLÉSIAS, J. C. R.; JATENE, M. B.; VERGINELLI, G.; JATENE, A. D. - Rotura cardiaca após infarto agudo do miocárdio (IAM): uma complicação passivel de correção cirúrgica? Rev. Bras. Cir. Cardiovasc., 8(4):272-281, 1993.

RESUMO: Foram estudados 9162 pacientes atendidos no INCOR, como diagnóstico de IAM, de janeiro de 1983 a dezembro de 1993 . Destes, $1,05 \%$ apresentaram rotura cardiaca de origem isquêmica como complicação do infarto miocárdico. A faixa etária média foi de 69,5 anos, predominando os pacientes de raça branca $(93,75 \%)$ e do sexo feminino $(55,3 \%)$. Os dados estudados incluíram história clínica, exames laboratoriais subsidiários, drogas utilizadas e achados cirúrgicos ou de necropsia. As roturas cardiacas foram classificadas, de acordo com a literatura, em agudas e sub-agudas. Observamos 72 casos de rotura miocárdica aguda com taxa de mortalidade de $98,6 \%$ e 24 casos de rotura sub-aguda com $41,6 \%$ de óbitos. Foram operados 4 pacientes na forma aguda e 15 na forma sub-aguda, resultando em $78,9 \%$ de sobrevida pósoperatória. Dos pacientes que receberam terapia trombolítica com sucesso $76,4 \%$ faleceram, enquanto que, dos pacientes tratados convencionalmente, esse número chegou a $86,1 \%$. Quando a terapia trombolítica foi administrada até 1 hora após o IAM, a mortalidade foi de $33,3 \%$, dentre 3 e 6 horas foi de $60 \%$ e após 6 horas foi de $100 \%$. A rotura ocorreu após 5 dias do IAM somente em $5,9 \%$ dos pacientes que receberam trombolíticos, enquanto que nos pacientes submetidos à terapêutica convencional esse índice elevou-se para $40,5 \%$. Concluímos pela gravidade e necessidade de atuação imediata nos pacientes com rotura cardíaca, mesmo nos casos sub-agudos, quando $30 \%$ dos pacientes com suspeita ecocardiográfica de expansão em área isquêmica transmural falecem. Nas roturas agudas, a situaçāo é dramática e a sobrevida está associada a fatores logisticos. Em condiçōes sub-agudas, entretanto, pode-se dispor de técnicas que dispensam suturas e circulaçāo extracorpórea, constituindo um importante recurso para o tratamento dessa grave complicação do IAM.

DESCRITORES: coração, rotura; infarto do miocárdio, cirurgia; agentes trombolíticos.

\section{INTRODUÇÃO}

A rotura cardíaca é uma grave complicação que acomete $1 \%$ a $4 \%$ dos pacientes admitidos no hospital, com infarto agudo do miocárdio (IAM) ${ }^{34}$. Calcula-se que ela seja responsável por $10 \%$ a $20 \%$ das causas de óbito após o IAM, sendo superada apenas pelo choque cardiogênico ${ }^{33}, 35$. A rotura da parede livre miocárdica tem incidência 3 a 10 vezes superior à rotura septal ou do músculo papilar 4,39 , e sua forma aguda é quase sempre fatal. Caracteriza-se pela rotura súbita e hemorragia maciça para a cavidade pericárdica, seguida de morte instantânea. Alguns pacientes, entretanto, podem sobreviver a esse evento inicial, quando a hemorragia para o pericárdio é lenta e repetitiva, podendo ser

Trabalho realizado no Instituto do Coração do Hospital das Clínicas da Faculdade de Medicina da Universidade de São Paulo.

São Paulo, SP, Brasil.

Recebido para publicação em dezembro de 1993.

- Do Instituto do Coração do Hospital das Clínicas da Faculdade de Medicina da Universidade de São Paulo.

Endereço para correspondência: Luís Alberto Dallan. Av. Dr. Enéas de Carvalho Aguiar, 44. Divisão Cirúrgica. 05403 São Paulo, SP, Brasil. 
DALLAN, L. A.; OLIVEIRA, S. A.; ABREU FILHO, C.; CABRAL, R. H.; JATENE, F. B.; PÊGO-FERNANDES, P. M.; IGLÉSIAS, J. C. R.; JATENE, M. B.; VERGINELLI, G.; JATENE, A. D. - Rotura cardiaca após infarto agudo do miocárdio (IAM): uma complicação passível de correçāo cirúrgica? Rev. Bras. Cir. Cardiovasc., 8(4):272-281, 1993.

contida pela formação de trombos entre o epicárdio e a cavidade pericárdica. Nesses casos ela é denominada sub-aguda, e o paciente pode sobreviver por horas ou dias, o que abre a possibilidade para eventual intervenção cirúrgica. Em casos especiais, a organização dos trombos no pericárdio pode desenvolver uma falsa cavidade ventricular esquerda, originando o chamado pseudo-aneurisma.

A introdução de agentes trombolíticos no tratamento do IAM tem alterado a evolução natural da rotura miocárdica. A rotura cardíaca ocorre apenas nos infartos com necrose transmural do miocárdio ${ }^{34}$, e a terapia trombolítica precoce tem se mostrado eficaz na limitação dessa necrose transmural ${ }^{15}$, contribuindo, portanto, para a redução das roturas miocárdicas. Por outro lado, resta saber se os trombolíticos administrados tardiamente após o IAM não elevam os riscos da rotura cardiaca. Essa dúvida baseia-se no fato da trombólise também promover hemorragia na área recém infartada e que o mecanismo da rotura cardíaca consiste na dissecção do sangue através das regiões de necrose transmural 15 .

Com o emprego rotineiro da ecocardiografia bidimensional nas unidades intensivas cardiológicas, tem-se sugerido que a dilatação regional e o afilamento do miocárdio recém-infartado podem ser elementos predictivos da rotura cardíaca ${ }^{36}$, o que assume extrema importância, na presteza da abordagem mais agressiva que essa complicação exige.

No que tange ao tratamento cirúrgico, temos observado e posto em prática novas técnicas sugeridas para a correção da rotura cardíaca, dispensando o emprego da circulação extracorpórea e simplificando o manuseio do vêntrículo recéminfartado.

Neste artigo, foi avaliada a incidência de rotura cardiaca dentre todos os pacientes atendidos no Instituto do Coração (INCOR) com diagnóstico de IAM, pelo período de 10 anos. Isso nos possibilitou compreender a localização da rotura e sua correlação com o número e grau das lesões nas artérias coronárias. Foi dada especial ênfase à diferenciação das roturas consideradas agudas e sub-agudas, e sua respectiva mortalidade. Procuramos, ainda, verificar a influência ou não do uso de agentes trombolíticos na prevenção ou desencadeamento da rotura cardíaca.

O exame clínico rigoroso de cada paciente, associado ao estudo ecocardiográfico e respectiva complementação cirúrgica, trouxeram resultados encorajadores para esse tipo de lesão que até há pouco tempo era considerada virtualmente fatal.

Nosso objetivo final busca verificar em que casos e sob que somatória de condutas a rotura cardíaca pode ser considerada passivel de correção cirúrgica com sucesso.

\section{CASUÍSTICA E MÉTODOS}

No período de janeiro de 1983 a dezembro de 1993 (10 anos), foram atendidos no INCOR 9162 pacientes com diagnóstico de IAM. Destes, 96 $(1,05 \%)$ apresentaram rotura cardiaca de origem isquêmica como complicação do infarto miocárdico. A faixa etária variou de 36 a 85 anos (média $=69,5$ anos). Noventa pacientes eram da raça branca, 4 amarela e 2 negra. Houve predomínio do sexo feminino (53 pacientes - $55,3 \%$ ), sobre o masculino (43 pacientes - 44,7\%) (Gráfico 1).

\section{Diagnóstico}

Os dados coletados dos pacientes incluíram sua história clínica, exames laboratoriais complementares e achados cirúrgicos ou de necropsia. Todos os pacientes foram observados quanto a idade, sexo, presença de IAM prévio, localização eletrocardiográfica do IAM, dor torácica prolongada, ocorrência de sincope, variaçōes nos niveis da pressão arterial e presença de pulso paradoxal. O registro do ECG permitiu também evidenciar alterações no ritmo cardiaco, bloqueios de ramo, bradicardia, dissociação eletromecânica, bloqueio átrio-ventricular ou fibrilação ventricular. O exame ecocardiográfico bidimensional foi realizado sempre que possível, na tentativa de se analisar a função contrátil das cavidades ventriculares e a presença de derrame pericárdico. Entretanto, a maioria dos pacientes já adentrou a unidade de emergência em situação crítica ou mesmo em parada cárdio-respiratória (PCR), o que impossibilitou sua investigação mais criteriosa, especialmente através do cateterismo cardíaco. Nesses casos deu-se preferência à simples pericardiocentese, que não só auxiliou no diagnós-

\section{GRÁFICO 1}

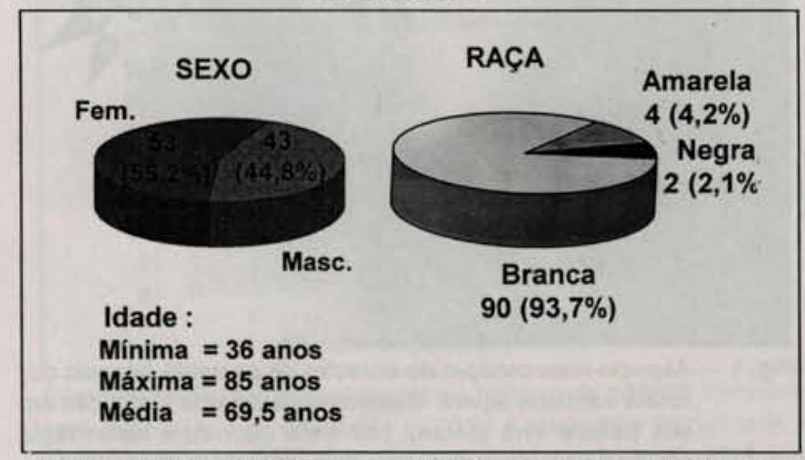

Distribuiçâo dos pacientes com rotura cardíaca após IAM quanto ao sexo, raça e faixa etária. 
DALLAN, L. A.; OLIVEIRA, S. A.; ABREU FILHO, C.; CABRAL, R. H.; JATENE, F. B.; PÊGO-FERNANDES, P. M.; IGLÉSIAS, J. C. R.; JATENE, M. B.; VERGINELLI, G.; JATENE, A. D. - Rotura cardiaca após infarto agudo do miocárdio (IAM): uma complicaçāo passível de correção cirúrgica? Rev. Bras. Cir. Cardiovasc., 8(4): 272-281, 1993.

tico, mas também contribuiu no alívio do tamponamento cardíaco.

O diagnóstico clínico foi estabelecido em 25 pacientes. Em 4 pacientes a rotura cardíaca foi constatada no intra-operatório, e nos demais 67 foi achada ou confirmada apenas na necropsia.

\section{Tipos de Rotura}

Dois tipos distintos de rotura da parede livre ventricular podem ser observados. A mais comum é a rotura aguda, súbita, com hemorragia maciça para a cavidade pericárdica, seguida de morte imediata (Figura 1). A forma sub-aguda caracteriza-se pela hemorragia mais lenta e repetitiva para o pericárdio, podendo levar horas ou dias. Uma outra manifestação da rotura ventricular sub-aguda implica na formação de um espaço organizado entre o epicárdio e o pericárdio, denominado pseudoaneurisma ventricular ${ }^{3}$ (Figuras 2 e 3 ).

\section{Reperfusão Coronária}

Dezessete $(17,7 \%)$ pacientes de nossa casuística haviam recebido terapia trombolítica através da administração endovenosa de estreptoquinase (SQ)

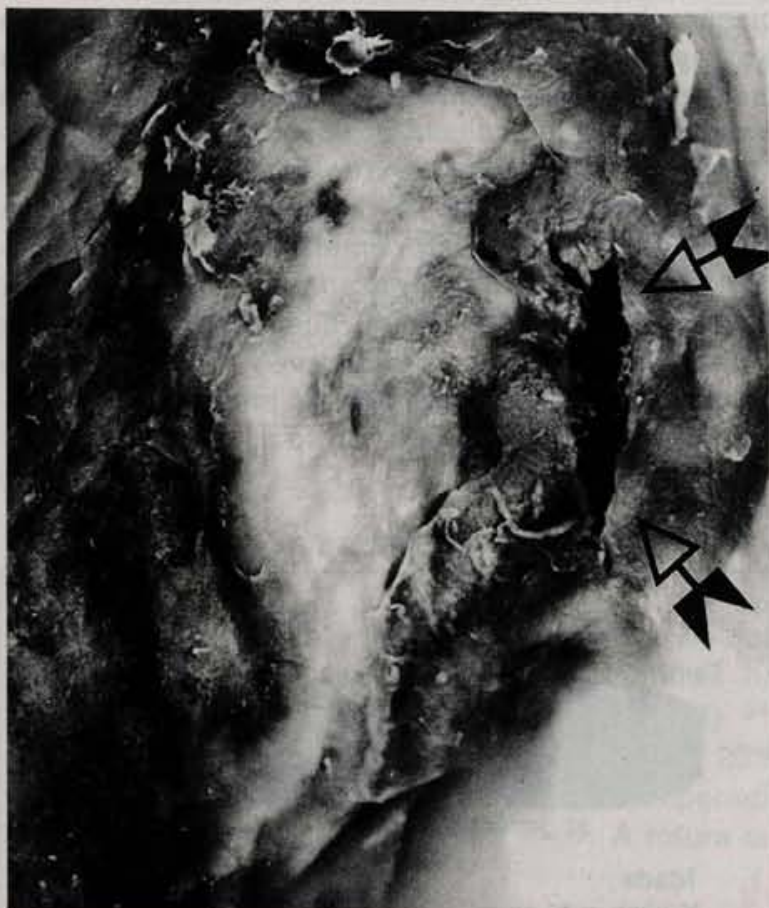

Fig. 1. Aspecto macroscópio do coração de paciente falecido por rotura cardiaca aguda. Destaca-se a externa laceração em sua parede livre (setas), por onde ocorreu a hemorragia maciça para a cavidade pericardiaca, seguida de tamponamento e morte instantânea.

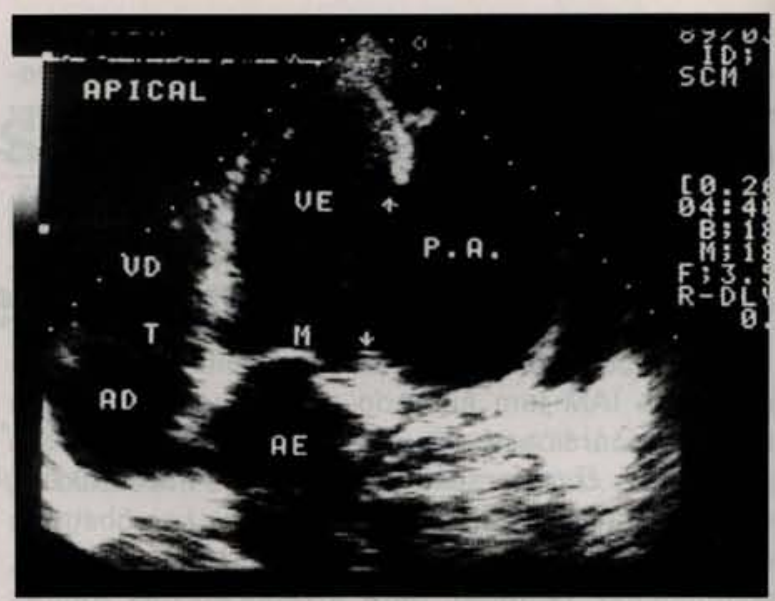

Fig. 2- Exame ecocardiográfico de paciente portador de pseudoaneurisma de VE. As cavidades cardiacas, assim como o colo (setas) e o pseudo-aneurisma (P. A.) são facilmente identificáveis através desse método.

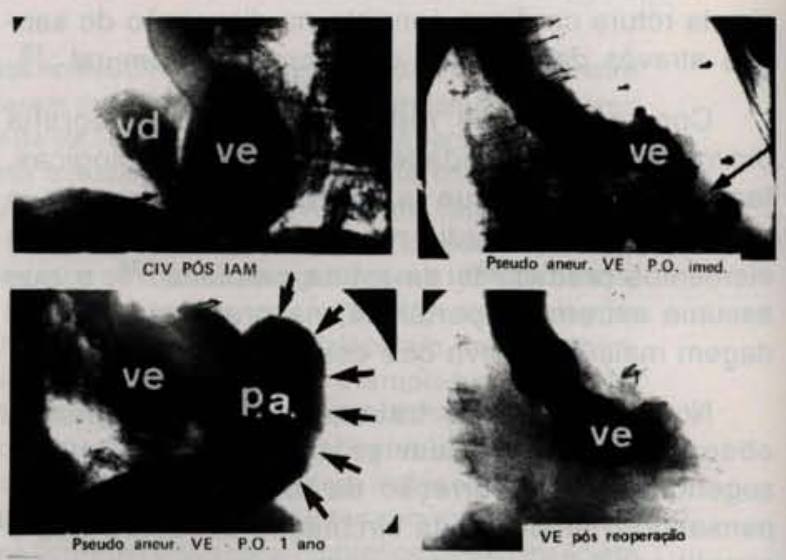

Fig. 3- Aspecto evolutivo cineangiográfico em paciente portador de comunicaçāo interventricular (CIV) pós IAM (quadro superior esquerdo), que apresentou pequena deiscência da ventriculorrafia esquerda (seta) após sua correção (quadro superior direito). O paciente retornou após 1 ano, com volumoso pseudo-aneurisma de VE (setas) (quadro inferior esquerdo), que foi corrigido após nova intervenção cirúrgica (quadro inferior direito).

ou rt-PA ("recombinant tissue-type Plasminogen Activator"), após o IAM. Três (17,6\%) receberam medicamentos até três horas do início dos sintomas, $5(29,4 \%)$ entre 3 e 6 horas e $9(52,9 \%)$ após 6 horas. Os demais $79(82,3 \%)$ não foram reperfundidos após o IAM.

\section{Tratamento Cirúrgico}

Apenas $19(19,8 \%)$ dos 96 pacientes foram operados para a correção da rotura miocárdica, sendo 4 em sua forma aguda e 15 na sub-aguda. Dentre os operados, $11(57,9 \%)$ tinham lesão em 
DALLAN, L. A.; OLIVEIRA, S. A.; ABREU FILHO, C.; CABRAL, R. H.; JATENE, F. B.; PÊGO-FERNANDES, P. M.; IGLÉSIAS, J. C. R.; JATENE, M. B.; VERGINELLI, G.; JATENE, A. D. - Rotura cardiaca após infarto agudo do miocárdio (IAM): uma complicação passível de correção cirúrgica? Rev. Bras. Cir. Cardiovasc., 8(4): 272-281, 1993.

apenas uma artéria coronária, $3(15,8 \%)$ em duas artérias, e $5(15,8 \%)$ em três artérias coronárias. Em apenas 3 pacientes se associou a revascularização do miocárdio. A técnica de correção incluiu o reforço da parede ventricular com feltro de Dacron ou pericárdio bovino em 16 pacientes. Em 3 pacientes, de casuística mais recente, foi empregado apenas o reforço de parede rota com tecido biológico e cola, sem utilização de suturas.

\section{Estudo Anatomopatológico}

Todos os pacientes falecidos foram necropsiados algumas horas após o evento. O local da rotura foi avaliado, assim como verificada presença de outras áreas de fibrose, e a severidade e a distribuição da aterosclerose coronária. Foram realizados cortes transversais desde o ápice até a base do coração, o que possibilitou, macroscopicamente, identificar a extensão do IAM. O exame histopatológico permitiu, também, estimar o tempo de evolução do IAM.

\section{Análise Estatística}

A análise de variança nos grupos foi realizada através do método do Qui-quadrado e do teste exato de Fisher. Foram considerados significativos valores iguais ou inferiores a 0,05 .

\section{Incidência, Localização e Mortalidade da Rotura Cardiaca}

Noventa e seis $(1,05 \%)$, dentre os pacientes atendidos no INCOR nos últimos ${ }^{\circ} \mathrm{dez}$ anos, tiveram rotura da parede livre do ventrículo esquerdo. A rotura ocorreu mais em mulheres $(55,2 \%)$ do que nos homens $(44,8 \%)$, com ampla predominância da raça branca $(93,7 \%)$.

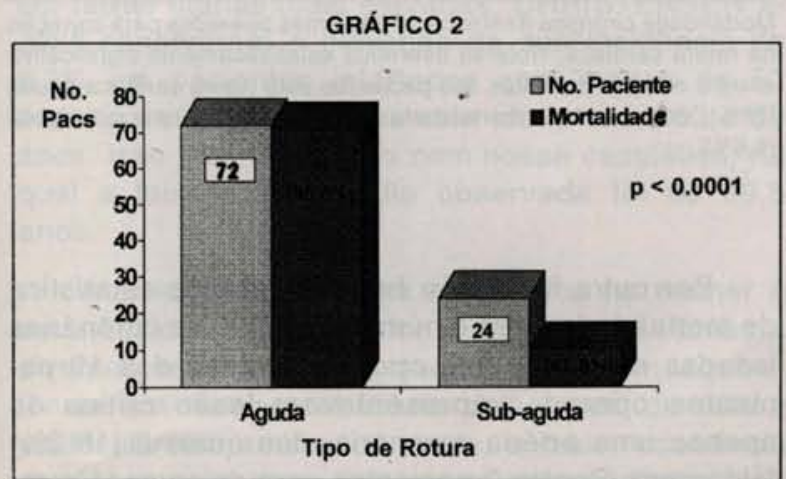

Distribuição dos pacientes com rotura cardiaca e respectiva mortalidade (operados e năo operados). Observa-se que praticamente todos os pacientes com rotura cardiaca aguda faleceram $(98,6 \%)$, contra $41,6 \%$ daqueles com rotura sub-aguda.
Observamos $72(75 \%)$ casos de rotura aguda da parede ventricular e $24(25 \%)$ casos em que ela foi sub-aguda. Pudemos constatar $71(98,6 \%)$ óbitos no grupo de pacientes com rotura aguda e 10 $(41,6 \%)$ óbitos dentre os pacientes com rotura cardíaca sub-aguda (Gráfico 2). No total, 81 (84,3\%) dos 96 pacientes estudados faleceram, com ampla prevalência dos portadores de rotura aguda $(p<0,0001)$.

A parede anterior do VE esteve comprometida em $40(41,7 \%)$ pacientes, a inferior em $12(12,5 \%)$ e a látero-posterior em $44(45,8 \%)$ (Tabela1).

O tempo mínimo decorrido entre o IAM e a rotura ventricular foi de 2 horas e 0 máximo de 11 dias, com média de 2,37 dias.

TABELA1

LOCALIZAÇĀO DA ROTURA DO VE

\begin{tabular}{lll}
\hline Anterior & $=$ & $40(41,7 \%)$ \\
Inferior & $=$ & $12(15,5 \%)$ \\
Látero-Posterior & $=$ & $44(45,8 \%)$ \\
\hline
\end{tabular}

Localizaçāo anatômica da parede ventricular esquerda em que ocorreu a rotura cardíaca.

\section{Doença Arterial Coronária}

Doença aterosclerótica de grau severo (obstrução de $70 \%$ ou mais da luz do vaso) comprometendo apenas uma artéria coronária foi observada em $37(38,5 \%)$ pacientes, dos quais $28(75,6 \%)$ faleceram. Em $15(15,6 \%)$ pacientes havia comprometimento bi-arterial, com $13(86,6 \%)$ óbitos. E nos 44 $(45,8 \%)$ restantes havia três ou mais artérias coronárias lesadas, dos quais $40(90,9 \%)$ vieram a falecer. O teste do Qui-quadrado $(p=0,168)$ não sugeriu diferença significativa de mortalidade no que se refere ao número de coronárias com lesão aterosclerótica severa (Gráfico 3 ).

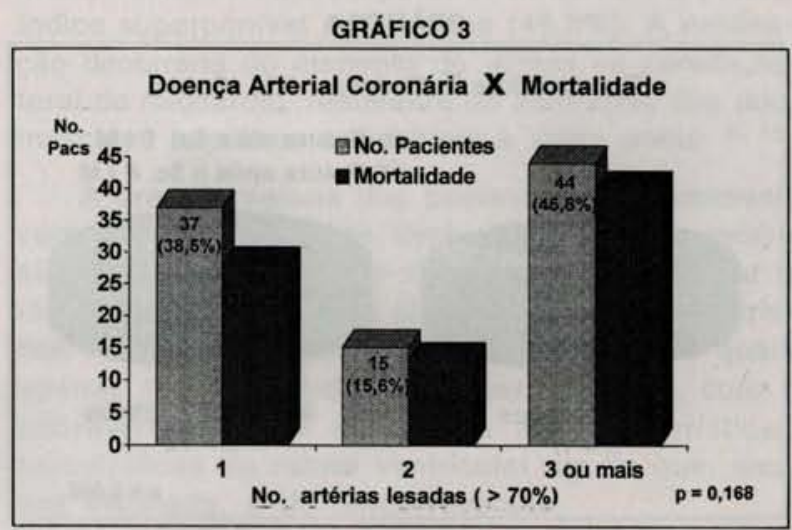

Distribuição dos pacientes quanto ao número de artérias coronárias com grau severo de obstruçāo (> 70\%) e respectiva mortalidade. O estudo estatístico $(p=0,168)$ não sugeriu diferença significativa entre os 3 grupos. 
DALLAN, L. A.; OLIVEIRA, S. A.; ABREU FILHO, C.; CABRAL, R. H.; JATENE, F. B.; PÊGO-FERNANDES, P. M.; IGLÉSIAS, J. C. R.; JATENE, M. B.; VERGINELLI, G.; JATENE, A. D. - Rotura cardiaca após infarto agudo do miocárdio (IAM): uma complicação passivel de correçäo cirúrgica? Rev. Bras. Cir. Cardiovasc., 8(4):272-281, 1993.

\section{Terapêutica Trombolítica e Rotura Cardíaca}

Dentre os 17 pacientes de nossa casuística que receberam algum tipo de trombolítico endovenoso, $13(76,5 \%)$ faleceram. Dos 79 pacientes não tratados com trombolíticos após o IAM, $68(86,1 \%)$ morreram. O teste exato de Fisher comparando a mortalidade dos pacientes com rotura ventricular que não receberam trombolítico versus os que receberam trombolítico não demonstrou diferença significativa $(p=0,243)$.

Entretanto, ao verificarmos a mortalidade após rotura cardíaca com o período após IAM em que o trombolítico foi administrado, observamos variação significativa. Dos 3 pacientes que receberam trombolítico até 3 horas do início dos sintomas, 1 $(33,3 \%)$ faleceu. Dentre 5 cujo trombolítico foi administrado entre 3 e 6 horas de dor, $3(60 \%)$ morreram. $E$ todos os 9 pacientes cujo trombolítico foi

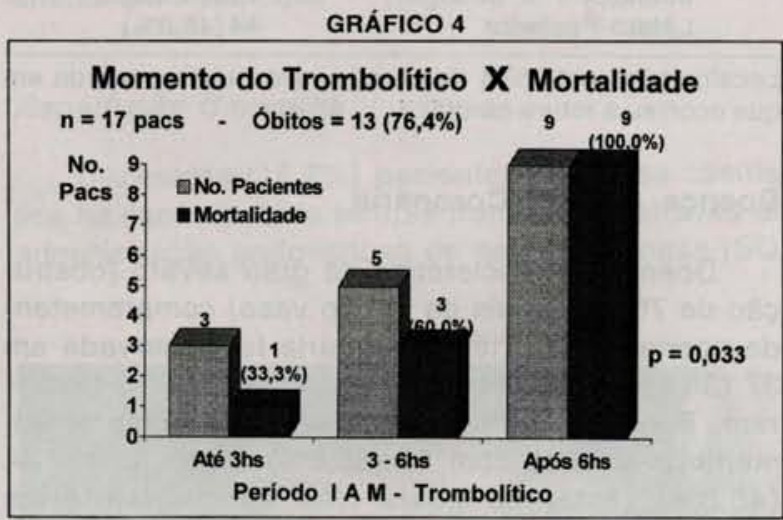

Distribuição dos 17 pacientes que receberam tromboliticos em periodos de 3, 6 ou mais horas após o IAM e que desenvolveram rotura cardiaca. Verificamos que a mortalidade se elevou progressivamente com a administraçăo mais tardia do trombolitico. $(p=0,033)$

\section{GRÁFICO 5}

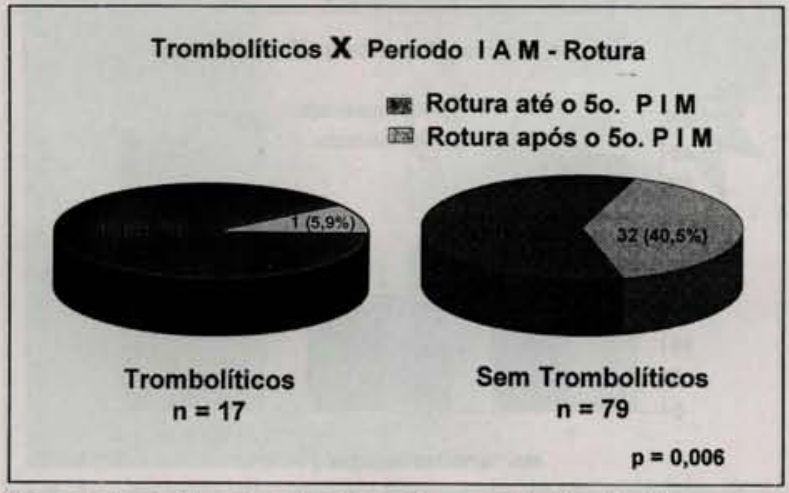

Periodo decorrido entre o IAM e a rotura ventricular. Verificamos que em $94,1 \%$ dos pacientes que receberam tromboliticos, a rotura ocorreu até o $5^{\circ}$ dia após o IAM. Nos pacientes sem trombolíticos esse indice caiu para $59,5 \%$. $(p=0,006)$ injetado após 6 horas faleceram $(p=0,033)$ (Gráfico 4).

A rotura cardíaca ocorreu nos primeiros 5 dias após o IAM em $16(94,1 \%)$ dos 17 pacientes que receberam trombolíticos e após o quinto dia em apenas $1(5,9 \%)$ paciente. Dentre os pacientes sem terapêutica trombolítica, a rotura se deu nos primeiros 5 dias em $47 .(59,5 \%)$ pacientes e foi tardia (após o quinto dia) nos $32(40,5 \%)$ restantes (Gráfico 5). A comparação estatística das proporçōes de rotura miocárdica nos dois grupos até o quinto dia após IAM, assim como no período complementar (tardia), revelou-se significativa $(p=0,006)$.

\section{Mortalidade Cirúrgica}

Dezenove $(19,8 \%)$ pacientes foram operados visando o reparo da rotura miocárdica, dos quais 4 $(21 \%)$ faleceram. Desse total, $15(78,9 \%)$ constituíam-se no grupo de rotura sub-aguda, dos quais 1 $(6,6 \%)$ faleceu. Dentre os $4(21,1 \%)$ pacientes do grupo agudo operados, $3(75 \%)$ faleceram. O teste exato de Fischer revelou diferença significativa de mortalidade entre esses grupos $(p=0,0016)$ (Gráfico 6).

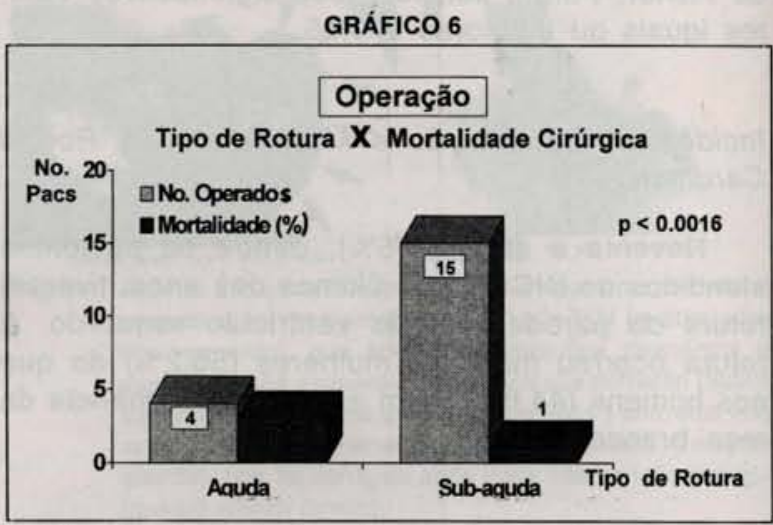

Mortalidade cirúrgica dentre os 19 pacientes operados para correção da rotura cardiaca. Nota-se diferença estatisticamente significativa entre o número de óbitos nos pacientes com rotura cardíaca aguda (75\%), comparado à mortalidade daqueles com rotura sub-aguda $(6,6 \%)$.

Por outro lado, não houve diferença estatística de mortalidade entre o número de artérias coronárias lesadas nos pacientes operados. Onze dos 19 pacientes operados apresentavam lesão crítica de apenas uma artéria coronária, dos quais $2(18,2 \%)$ faleceram. Dentre 3 pacientes com dois vasos comprometidos, apenas $1(33,3 \%)$ faleceu. $E$ nos 5 demais pacientes com três ou mais artérias coronárias lesadas, $1(20 \%)$ faleceu $(p=1,000)$. 
DALLAN, L. A.; OLIVEIRA, S. A.; ABREU FILHO, C.; CABRAL, R. H.; JATENE, F. B.; PÊGO-FERNANDES, P. M.; IGLÉSIAS, J. C. R.; JATENE, M. B.; VERGINELLI, G.; JATENE, A. D. - Rotura cardiaca após infarto agudo do miocárdio (IAM): uma complicaçāo passivel de correçāo cirúrgica? Rev. Bras. Cir. Cardiovasc., 8(4): 272-281, 1993.

\section{COMENTÁRIOS}

A rotura cardíaca pós IAM foi inicialmente descrita por HARVEY ${ }^{14}$, em 1647. Já em 1769, MORGANI 25 relatou 10 casos dessa patologia. Passados mais de 200 anos, BATTS et alii ${ }^{4}$ coletaram na literatura mais de 1200 casos de rotura cardiaca, sendo 20 de sua própria casuística.

A incidência de rotura cardíaca varia de $4 \%$ a $20 \%$ na experiência de diversos autores $19,26,32$. Entretanto, existem relatos de sua evidência em até $38 \%$ das necropsias de pacientes falecidos de IAM ${ }^{15}$. Com o advento das unidades de tratamento intensivo coronariano, o diagnóstico de rotura miocárdica tem sido mais freqüente, não só pelo avanço nos métodos diagnósticos, mas também pela menor mortalidade imediata decorrente das outras complicações do IAM ${ }^{35}$.

A rotura da parede livre ventricular atualmente superou as arritmias constituindo a segunda causa de morte em pacientes na fase aguda do IM 28 . Trata-se da mais grave complicação mecânica do $I A M$, sendo 3 a 10 vezes mais freqüente do que a rotura do músculo papilar de valva mitral ou do septo interventricular 8 .

A rotura cardíaca ocorre em regiōes de completa necrose miocárdica transmural, geralmente após a transformação do IAM isquêmico em hemorrágico. Normalmente ela coincide com extensa hemorragia localizada na região da necrose, onde a isquemia foi severa o suficiente para causar necrose da microvasculatura 7,15 . A rotura completa da parede cardiaca pode ter seu início tanto na superfície endocárdica, quanto em meio às fibras do miocárdio, dissecando a zona do IM até atingir o epicárdio ${ }^{15}$. Entretanto, BATTS et alii ${ }^{5}$ consideram a possibilidade dessa rotura ocorrer na zona de transição entre o miocárdio infartado e o saudável, local onde as forças de cisalhamento são mais intensas 11 .

A rotura cardíaca ocorre com maior freqüência em faixas etárias mais elevadas. CHRISTENSEN et alii ${ }^{6}$ observaram a elevação de sua incidência de $0,3 \%$ em pacientes infartados com menos de 50 anos, para 2,2\% naqueles com idade entre 60 e 69 anos. Isso vem de acordo com nossa casuística, na qual a faixa etária média observada foi de 69,5 anos.

A incidência de rotura miocárdica na mulher é reconhecidamente superior à do homem. Embora diversos relatos de literatura demonstrem equivalência em número absoluto dos casos de rotura ventricular em ambos os sexos, a maior ocorrência de IAM no sexo masculino reforça essa evidência ${ }^{9,28}$. Pudemos corroborar esses dados em nossa casuística, dada a maior incidência de rotura em mulheres $(55,2 \%)$.
A presença de hipertensão arterial sistêmica após o IAM tem sido associada à maior incidência de rotura cardíaca, principalmente nos corações de tamanho normal 4,40 . Seu mecanismo de atuação seria o stress mecânico sobre a parede enfraquecida pelo IM, decorrente da elevada força de contração do tecido miocárdico normal adjacente e associada à elevação da pressão intracavitária durante a sístole.

Entretanto, nos pacientes com hipertensão arterial sistêmica crônica, a rotura cardíaca tende a ser menos freqüente. Isso porque a presença da hipertrofia ventricular, comum nessas pacientes, constitui um fator natural de proteção 5 . A menor freqüência de hipertrofia ventricular nas mulheres pode justificar a maior tendência de rotura cardíaca nesse sexo.

A rotura cardiaca acomete mais freqüentemente pacientes no primeiro episódio do IAM, muitos deles sem episódios anteriores de dor precordial ${ }^{16}$, MANN \& ROBERTS ${ }^{22}$, estudando 138 pacientes com rotura da parede livre do VE, observaram ausência de infartos antigos em $87 \%$ dos pacientes.

Essas evidências contrastam com o grau de comprometimento arterial coronário, uma vez que as roturas miocárdicas incidem preferencialmente em pacientes multiarteriais. BATTS et alii ${ }^{5}$ analisando 100 necropsias pós rotura ventricular, observaram comprometimento de três ou mais artérias em $40 \%$ das mesmas. Em nossa casuística, o índice de comprometimento arterial múltiplo também predominou, atingindo $45,8 \%$ dos casos.

Apesar de inúmeros relatos sugerirem que a parede miocárdica anterior seja a mais susceptivel de rotura $(5,26)$, o mesmo não foi observado por outros autores. PADRÓ et alii ${ }^{30}$ advogam que a rotura cardíaca pode ocorrer igualmente em qualquer região do VE. BATTS et alii ${ }^{5}$ observaram maior acometimento na parede lateral do VE $(44 \%)$, índice superponivel aos nossos $(45,8 \%)$. A explicação decorreria do aumento do stress na parede lateral do miocárdio, resultante da contração dos dois músculos papilares adjacentes à valva mitral 4,19 .

A grande maioria dos pacientes que desenvolvem rotura da parede livre do miocárdio morre subitamente, sem tempo hábil para se completar o diagnóstico ${ }^{19}$. Pudemos observar em nossa casuística $72(75 \%)$ pacientes nessa situação, dos quais apenas $4(5,6 \%)$ chegaram a ser operados, com 1 sobrevivente. Isso demonstra as características catastróficas da rotura ventricular aguda que, uma vez instalada, é de difícil reversão.

Entretanto, num considerável número de pacientes, a rotura se instala de forma sub-aguda, o que possibilita sua identificação e abre espaço para 
DALLAN, L. A.; OLIVEIRA, S. A.; ABREU FILHO, C.; CABRAL, R. H.; JATENE, F. B.; PÊGO-FERNANDES, P. M.; IGLÉSIAS, J. C. R.; JATENE, M. B.; VERGINELLI, G.; JATENE, A. D. - Rotura cardiaca após infarto agudo do miocárdio (IAM): uma complicação passivel de correção cirúrgica? Rev. Bras. Cir. Cardiovasc., 8(4): 272-281, 1993.

eventual tratamento cirúrgico. A incidência exata de rotura sub-aguda do miocárdio näo está bem estabelecida. LOPEZ-SENDON et alii ${ }^{21}$ estimam em $30 \%$ os casos em que a rotura ventricular pós IAM não se segue de morte súbita. Pudemos observar, dentre nossos pacientes, $24(25 \%)$ pacientes com rotura sub-aguda, todos encaminhados para tratamento cirúrgico.

O diagnóstico de rotura ventricular sub-aguda requer uma decisão cirúrgica. Segundo LOPEZSENDON et alii ${ }^{21}, 76 \%$ dos pacientes operados nessa situação sobreviveram ao procedimento, e $48,5 \%$ deles apresentavam sobrevida após seguimento médio de 30 meses. Em nossa casuística, $93,4 \%$ dos pacientes com rotura sub-aguda obtiveram alta hospitalar, o que enfatiza a necessidade do diagnóstico precoce dessa complicação do IAM.

Não existe um quadro clínico patognomônico da rotura ventricular sub-aguda. As manifestações clínicas iniciais mais freqüentes incluem desde a hipotensão arterial isolada, até a dor torácica intensa pouco responsiva a opiáceos. Podem também ocorrer episódios de sincope ou dissociação eletromecânica transitória. Nessa fase, o diagnóstico pode ser facilitado através de exames complementares como o ECG, ecodopplercardiografia e pela monitorização invasiva através do cateter de SwanGanz.

As alterações eletrocardiográficas iniciais são inespecíficas, em geral com características do IM transmural, supradesnivelamento de ST. baixa amplitude do complexo QRS, inversões de onda T e distúrbios de condução intraventricular ${ }^{24}$.

O cateter de Swan-Ganz pode evidenciar a elevação da pressão atrial direita, com a equalização diastólica das pressões de átrio direito, artéria pulmonar e capilar pulmonar. Deve-se, entretanto, lembrar que medidas semelhantes podem ocorrer na disfunção ventricular direita.

Atualmente, ganha grande destaque a monitorização ecocardiográfica bidimensional no paciente infartado. A expansão da parede miocárdica infartada constitue um sinal potencialmente predictivo da rotura cardiaca, e sua detecção pelo ecocardiograma pode alterá-la antes que se consume a completa rotura do miocárdio. Numa fase mais avançada, já na presença de efusão pericárdica, os sinais e sintomas de tamponamento cardíaco passam a ser preponderantes.

O ecocardiograma transtorácico e esofágico detectam, com clareza, alterações na motilidade das contrações cardíacas e sinais de compressão das câmaras direitas, especialmente do ventrículo direito $1,2,12,31$. O sinal ecocardiográfico patognomônico, entretanto, consiste na visibilização direta da rotura da parede ventricular 10,37 .
A pericardiocentese, demonstrando presença de sangue na cavidade pericárdica, tem sido considerada como critério de elevada confiabilidade no diagnóstico de rotura ventricular sub-aguda 18,30 . 0 alívio que proporciona ao tamponamento cardíaco também tem seu valor terapêutico. Por outro lado, a evidência de líqüido pericárdico de aspecto seroso, nos pacientes com suspeita ecocardiográfica de tamponamento cardíaco, afasta o diagnóstico de rotura miocárdica 21.

A terapia trombolítica (SQ, rt-PA), associada à angioplastia transluminal coronária (ATC), tem sido empregada de rotina na fase aguda do IAM, reduzindo sua expansão. Com isso a mortalidade dessa fase do IAM tem sido significativamente reduzida, passando de $50 \%$ para $26 \% \quad 28$. O trombolítico administrado até 6 horas do início dos sintomas reduz a possibilidade da progressão transmural do IAM, o que indiretamente também previne a rotura miocárdica.

Entretanto, existem evidências de que a terapêutica trombolítica tardia (acima de 6 horas) possa elevar o número de roturas miocárdicas ${ }^{6}$. Isso se deve à dissecção hemorrágica transmural ocasionada pela reperfusão da microvasculatura de uma área de necrose extensa ${ }^{7}$ e pelo retardamento da cicatrização miocárdica 36,37 .

Com a introdução no INCOR, a partir de 1989 , de agentes trombolíticos para pacientes na fase aguda do IM, pudemos constatar mudança na evolução natural da rotura miocárdica. Observamos menor mortalidade por rotura cardiaca nos pacientes infartados que receberam trombolíticos $(76,4 \%)$, do que nos que não receberam a droga $(86,1 \%)$.

Entretanto, ao analisarmos com maior profundidade apenas os pacientes que receberam trombolíticos, verificamos maior mortalidade estatisticamente significativa) nos reperfundidos após 6 horas do início dos sintomas, do que nos pacientes reperfundidos precocemente.

Além disso, $94,1 \%$ de nossos pacientes que receberam agentes trombolíticos tardiamente apresentaram rotura ventricular nos primeiros 5 dias do IAM, e apenas $5,9 \%$ após essa fase. Esses resultados contrastam com o dos pacientes tratados convencionalmente, dos quais $40,5 \%$ romperam o miocárdio após o quinto dia de IM. Essas evidências deixam transparecer que 0 trombolítico administrado após 6 horas de IAM pode elevar o índice de roturas precoces.

A revascularização miocárdica tardia, à semeIhança do agente trombolítico, pode igualmente transformar IAM isquêmico em hemorrágico, podendo predispor à rotura miocárdica $7,17,23,38$. 
DALLAN, L. A.; OLIVEIRA, S. A.; ABREU FILHO, C.; CABRAL, R. H.; JATENE, F. B.; PÊGO-FERNANDES, P. M.; IGLÉSIAS, J. C. R.; JATENE, M. B.; VERGINELLI, G.; JATENE, A. D. - Rotura cardiaca após infarto agudo do miocárdio (IAM): uma complicaçāo passivel de correção cirúrgica? Rev. Bras. Cir. Cardiovasc., 8(4): 272-281, 1993.

O tratamento cirúrgico da rotura sub-aguda do ventrículo esquerdo vem trazendo resultados gratificantes. A sobrevida hospitalar de mais de $90 \%$ de nossos pacientes, operados nessa condição, estimula esse procedimento.

Novas técnicas, que dispensam circulação extracorpórea e evitam a infartectomia, vêm sendo utilizadas nos últimos anos ${ }^{13}$. Já em 1983, NUNEZ et alii ${ }^{29}$ propuseram o tamponamento da área rota com sutura de retalho de Teflon fixado ao miocárdio normal, tendo obtido $57 \%$ de sobrevida. Em 1988 , PADRÓ et alii ${ }^{30}$ descreveram técnica semelhante, porém com o emprego de cola biológica (cianoacrilato) ao invés da sutura com Polipropileno. Os autores relatam o tratamento de 13 pacientes portadores de rotura sub-aguda de ventrículo es- querdo com essa técnica, sem mortalidade.

Concluímos pela gravidade e necessidade de atuação imediata nesses pacientes. A suspeita ecocardiográfica de expansão em área isquêmica transmural constitui um alerta ao cirurgião, uma vez que mesmo nos casos sub-agudos, cerca de $30 \%$ dos pacientes com esses sinais falecem em menos de uma hora. Nas roturas agudas a situação é dramática e a sobrevida está associada a fatores logísticos. Em condiçōes sub-agudas, entretanto, tem-se dado recente ênfase a técnicas que dispensam suturas e circulação extracorpórea. O tamponamento da rotura com retalhos de tecido e cola biológica, como acima citado, vem se constituindo num importante recurso para o tratamento dessa grave complicação do IAM.

RBCCV 44205-224

DALLAN, L. A.; OLIVEIRA, S. A.; ABREU FILHO, C.; CABRAL, R. H.; JATENE, F. B.; PÊGO-FERNANDES, P. M.; IGLESIAS, J. C. R.; JATENE, M. B.; VERGINELLI, G.; JATENE, A. D. - Cardiac rupture after acute myocardial infarction (AMI): may it have a surgical repair? Rev. Bras. Cir. Cardiovasc., 8(4):272-281, 1993.

ABSTRACT: PURPOSE: Analise the incidence of cardiac rupture within the patients received in our hospital with the diagnosis of AMI in a period of 10 years and try to identify cases when the cardiac rupture can be submitted to a successful approach. METHODS: 9162 patients were received by INCOR with the diagnosis of $\mathrm{AMI}$ in the period from january 1983 to december 1993 . From these patients $1.05 \%$ had cardiac rupture as an ischaemic complication of the myocardium infarction. The average of age was 69.5 years and showing a predominance of white people $(93.75 \%)$ and female sex $(55.3 \%)$. Data from patients include clinical history, complementary investigations, drugs used in the treatment and surgical or anatomopathological findings. The cardiac ruptures were classified as acute and sub-acute, according to literature. RESULTS: We found 72 cases of acute myocardial rupture with a mortality rate of $98.6 \%$ and 24 cases of sub-acute myocardial rupture with $41.6 \%$ of deaths. Four patients were operated on acute rupture and 15 patients were operated on subacute rupture. The post surgery survival was $78.9 \%$. The patients who had a successful thrombolitic therapy, $76.4 \%$ died while the others who received routine therapy, $86.1 \%$ passed away. Once the thrombolitic therapy was given until an hour the mortality was 33.3\%; from 3 to 6 hours it was $60 \%$ and after 6 hours it was $100 \%$. When it turns to the lenght of time of the onset of the cardiac rupture after IAM treatment, it happend after 5 days just in $5.9 \%$ of the patients who received trombolitic agents but in $40.5 \%$ of the patients with the routine therapy. CONCLUSIONS: We recognized the importance and the necessity of a immediate action for the patient with cardiac rupture even in sub-acute cases when $30 \%$ of the patients with an echographic probability of having a progression of the transmural ischaemic area die. In acute ruptures the situation is dramatic and survical depends on logistic factors. In sub-acute conditions however, new sutureless techniques without using extra-corporeal circulation are now available and they will have a huge importance in the treatment of this extremely serious complication of AMI.

DESCRIPTORS: myocardial infarction, surgery; thrombolitic agents.

\section{REFERÊNCIAS BIBLIOGRÁFICAS}

1 AKASAKA, T.: YOSHIKAWA, J.: YOSHIDA, K. Ventricular free wall rupture following acute myocardial infarction: a two dimensional echocar-diographic assessment. J. Cardiol., 18: 89-97, 1988.
R.; MACEDO, M.; RIBEIRO, C. - Rotura da parede livre do ventrículo esquerdo na fase aguda do enfarte do miocárdio operada com sucesso. Rev. Port. Cardiol., 6: 315-322, 1987.

3 BALAKUMARAN, K.; VERBAAN, C. J.; ESSED, C. E. - Ventricular free wall rupture: sudden subacute slow sealed and stabilized varieties. Eur. Heart J., 5: 282-293, 1984 
DALLAN, L. A.; OLIVEIRA, S. A.; ABREU FILHO, C.; CABRAL, R. H.; JATENE, F. B.; PÊGO-FERNANDES, P. M.; IGLÉSIAS, J. C. R.; JATENE, M. B.; VERGINELLI, G.; JATENE, A. D. - Rotura cardiaca após infarto agudo do miocárdio (IAM): uma complicaçăo passível de correção cirúrgica? Rev. Bras. Cir. Cardiovasc., 8(4): 272-281, 1993.

4 BATES, R. J.; BEUTLER, S.; RESNEKOV, L.; ANAGNOSTOPOULOS, C. E. - Cardiac rupture: challange in diagnosis and management. Am. J. Cardiol., 40: 429-437, 1977.

5 BATTS, K.; ACKERMANN, D. M.; EDWARDS, W. D. Postinfarction rupture of the left ventricular free wall: clinicopathologic correlates in 100 consecutive autopsy cases. Human Pathol., 21: 530-535, 1990.

6 CHRISTENSEN, D. J.; FORD, M.; READING, J.; CASTLE, C. H. - Effect of hipertension on myocardial rupture after acute myocardial infarction. Chest., 72: 618-622, 1977.

7 DALLAN, L. A. O.; OLIVEIRA, S. A.; LAVITOLA, P. VERGINELLI, G.; JATENE, A. D. - Infarto hemorrágico conseqũente à reperfusão miocárdica. Relato de quatro casos. Arq. Bras. Cardiol., 54: 127-132, 1990.

DALLAN, L. A.; OLIVEIRA, S. A.; RAMIRES, J. A. F.; SABINO NETO, A.; VERGINELLI, G.; JATENE, A. D. - Tratamento cirúrgico da comunicação interventricular pós infarto agudo do miocárdio. Rev. Bras. Cir. Cardiovasc., 4: 64-74, 1989.

9

DELLBORG, M.; HELD, P.; SWEDBERG, K. - Rupture of the myocardium: occurrence and risk factors. $\mathrm{Br}$. Heart J., 54: 11-16, 1985.

DESOUTTER, P.; HALPHEN, C.; HAIAT, R. - Two dimension echocardiographic visualization of free ventricular wall rupture in acute anterior myocardial infarction. Am. Heart J., 108: 1360-1367, 1984.

11 ELIOT, R. S. \& EDWARDS, J. E. - Pathology of coronary atherosclerosis and its complications. In Hurst JW, Logue RB, Schlant RC: The Heart: arteries and veins. 4 ed. New York, NY. Mc Graw-Hill, 1978. p. 1121. 1134.

ENNIX Jr, C. L.; ECKER, R. R.; IVERSON, L. I. - Early detection and management of left ventricular free rupture during acute myocardial infarction. $A m$. J. Cardiol., 63: 151-152, 1989.

TZGIBBON, G. M.; HOOPER, G. D.; HEGGTVEIT, H. A. - Successful surgical treatment of postinfarction external cardiac rupture. J. Thorac. Cardiovasc. Surg., 63: 622-630, 1972.

HARVEY, W. - De Circulatio Sanguinis. Exercit 3. Citado por Morgagni GB In: The seats and causes of diseases. Traduçāo Benjamin Alexander. London, Letter 27, p. 830,1769

HONAN, M. B.; HARRELL, F. E.; REIMER, K. A.; CALIFF, R. M.; MARK, D. B.; PRYOR, D. B.; HLATKY, M. A. - Cardiac rupture, mortality and the timing of thrombolytic therapy: a meta-analysis. J.A. C. C., 16: $359-367,1990$

ISHIBASHI-UEDA, H.; IMAKITA, M.; FUJITA, $\mathrm{H}_{\text {; }}$ KATSURAGI, M.; YUTANI, C. - Cardiac rupture complicating hemorrhagic infarction after intracoronary thrombolysis. Acta Path. Jpn., 42: 504507,1992

17 KAO, K. J.; HACKEL, D. B.; KONG, Y. - Hemorrhagic myocardialinfarction after streptoquinase treatment for acute coronary thrombosis. Arch. Pathol. Lab. Med., 108: 121-124, 1984.

18 KENDALL, R. W. \& DE WOOD, M. A. - Postinfarction cardiac rupture: surgical success and review of the literature. Ann. Thorac. Surg., 25: 311-315, 1978.

19 LEONE, A. - Are we able to prevent death due to postinfarction cardiac rupture by early diagnosis and surgical treatment? Jpn. Heart J., 32: 635-644, 1991.

20 LEVENE, A. - Spontaneous rupture of the heart. Br. Heart J., 22: 660-670, 1960.

21 LÓPEZ-SENDQ́N, J.; GONZALES, A.; SÁ, E. L.; COMACANELLA, I.; ROLDAN, I.; DOMINGUEZ, F.; MAQUEDA, I.; JADRAQUE, L. M. - Diagnosis of subacute ventricular wall rupture after acute myocardial infarction: sensitivity snd specificity of clinical, hemodynamic and echocardiographic criteria - J. A. C. C., 19: 1145-1153, 1992.

22 MANN, J. M. \& ROBERTS, W. C. - Rupture of the left ventricular free wall during acute myocardial infarction: analysis of 138 necropsy patients and comparison with 50 necropsy patients with acute myocardial infarction without rupture. Am. J. Cardiol., 62: 847$859,1988$.

23 MATHEY, D. G.; SCHOFER, J.; KUCK, K-H; BEIL, U.; KLOPPEL, G. - Transmural, haemorrhagic myocardial infarction after intracoronary streptoquinase: clinical, angiographic, and necropsy findings. Br. Heart J., 48: 546-551, 1982.

24 MEURS, A. A. H.; VOS, A. K.; VERHEY, J. B.; GERBRANDY, J. - Electrocardiogram during cardiac rupture by myocardial infarction. Br. Heart J., 32: 232-236, 1970.

MORGAGNI, J. B. - The seats and causes of diseases investigated by anatomy. vol 1 . Tradução de B. Alexander. London, Millar A. Cadell T. 1769; p. 811 . 834.

NAEMIN, F.; DE LA MAZA, L. M.; ROBBINS, S. L. Cardiac rupture during myocardial infarction: a review of 44 cases. Circulation, 45: 1231-1939, 1972.

27 NAKAJIMA, H. \& EDWARDS, J. E. - Factors favoring certain complications of acute myocardial infarction. Minnesota Med., 40: 291-295, 1985.

28 NAKAMURA, F.; MINAMINO, T.; HIGASHINO, Y.; ITO, H.; FUJII, K.; FUJITA, T.; NAGANO, M.; HIGAKI, J.; OGIHARA, T. - Cardiac free wall rupture in acute myocardial infarction: ameliorative effect of coronary reperfusion. Clin. Cardiol., 15: 244-250, 1992.

29 NUNEZ, L.; DE LA LLANA, R.; LÓPEZ-SENDÓM, J.; 
DALLAN, L. A.; OLIVEIRA, S. A.; ABREU FILHO, C.; CABRAL, R. H.; JATENE, F. B.; PÊGO-FERNANDES, P. M.; IGLÉSIAS, J. C. R.; JATENE, M. B.; VERGINELLI, G.; JATENE, A. D. - Rotura cardiaca após infarto agudo do miocárdio (IAM): uma complicação passivel de correção cirúrgica? Rev. Bras. Cir. Cardiovasc., 8(4): 272-281, 1993.

COMA, I.; GIL AGUADO, M.; LARREA, J. L. Diagnosis and treatment of subacute free wall ventricular rupture after infarction. Ann. Thorac. Surg., 35: 525-529, 1983.

PADRÓ, J. M.; MESA, J. M.; SILVESTRE, J.; LARREA, J. L.; CARALPS, J. M.; CERRÓN, F.; ARIS, A. Subacute cardiac rupture: repair with a sutureless technique. Ann. Thorac. Surg., 55: 20-24, 1993.

31 PAPPAS, P. J.; CERNAIANU, A. C.; BALDINO, W. A. CILLEY Jr, J. H.; DELROSSI, A. J. - Ventricular freewall rupture after myocardial infarction: treatment and outcome. Chest, 99: 892-895, 1991.

PENTER, P.; GERBAUX, A.; BLANC, J. J.; MORIN, J. F.; JULIENNE, J. L. - Myocardial infarction and rupture of the heart: a macroscopic pathologic study: $A m$. Heart J., 93: 302-331, 1977.

33 PERDIGĀO, C.; LÓPEZ-SENDÓN, J.; FROUFE, J. Causas de morte no enfarte agudo do miocárdio: estudio multicéntrico lbérico. Rev. Port. Cardiol., 7: $31-35,1988$

RASMUSSEN, S.; LETH, A.; KJOLLER, E.; PEDERSEN, A. - Cardiac rupture in acute myocardial infarction. Acta Med. Scand., 205: 11-16, 1979.
REDDY, S. G.; ROBERTS, W. C. - Frequency of rupture of the left ventricular free wall or ventricular septum among necropsy cases of fatal acute myocardial infarction since introduction of coronary care units. Am. J. Cardiol., 63: 906-911, 1989.

SCHUSTER, E. H. \& BULKLEY, B. H. - Expansion of transmural myocardial infarction: a pathophysiologic factor in cardiac rupture. Circulation, 60: 1532-1538, 1979.

37 SHAPIRA, I.; ISAKOV, A.; BURKE, M.; ALMOG, C. Cardiac rupture in patients with acute myocardial infarction. Chest., 92: 219-223, 1987.

TWINDALE, N.; HENRY, L.; MORPHETT, A.; TONKIN, A. M. - Hemorrhagic myocardial infarction complicated by free wall-rupture: a case associated with unusual clinical features following intravenous thrombolitic therapy. Aust. N. Z. J. Med., 19: 138-140, 1989.

39 VAN TASSEL, R. A. \& EDWARDS, J. E. - Rupture of heart complicating myocardial infarction: analysis of 40 cases including nine examples of left ventricular false aneurysm. Chest, 61: 104-116, 1972.

WESSLER, S.; ZOLL, P. M.; SCHLESINGER, M. J. The pathogenesis of spontaneous cardiac rupture. Circulation, 6: 334-351, 1952. 